\title{
Mejora de las competencias gracias a la implantación de aprendizaje basado en proyectos en acuicultura Improve of competences through project-based learning in aquaculture
}

\author{
David S. Peñaranda, Ignacio Jauralde-García, Ana Tomás-Vidal, Miguel Jover-Cerdá, Silvia Martínez-Llorens \\ dasncpea@upv.es, igjaugar@upv.es, atomasv@dca.upv.es,mjover@dca.upv.es, silmarll@dca.upv.es \\ ${ }^{1}$ Departamento de Ciencia Animal \\ Universitat Politècnica de València \\ Valencia, España
}

\begin{abstract}
Resumen- En el presente trabajo se muestra la experiencia de la aplicación del Aprendizaje Basada en proyectos (ABP) en la asignatura "Diseño y Gestión de instalaciones Piscícolas" de la titulación del Máster interuniversitario de Acuicultura de la Universitat Politécnica de València. El ABP consistió en el desarrollo de un proyecto de una piscifactoría, en el cual los alumnos debían integrar los conocimientos adquiridos en las asignaturas previas, permitiendo una visión más multidisciplinar de la asignatura y mejor adquisición de las competencias. Tanto desde el punto de vista del alumnado como del profesor, la implantación del ABP permitió un mayor desarrollo de las competencias, siendo la metodología docente valorada positivamente. Además, se mejoró la participación del alumnado en las actividades propuestas, dando como resultado una mejor calificando académica final. No obstante, se deben de mejorar algunos aspectos como es la planificación del tiempo y consolidar relaciones de integración o la interacción entre diferentes disciplinas/asignaturas de manera que tengan una mejor integración en el proyecto.
\end{abstract}

Palabras clave: Aprendizaje basado en proyectos, multidisciplinar, competencias

Abstract- This manuscript shows the experience of the implantation of a Project-Based Learning (ABP) in the subject "Design and Management of Fish Farming Facilities" in the Interuniversity Master's Degree in Aquaculture at the Universitat Politécnica de València. The ABP consisted in the development of a project for a fish farm, in which students had to integrate the knowledge acquired in previous subjects, allowing a more multidisciplinary learning and better acquisition of competences. Both from point of view, students and teachers, the implementation of ABP has allowed a greater development of competences, being the teaching methodology positively evaluated. In addition, student participation in the proposed activities was improved, resulting in a better final academic grade. However, some aspects should be improved, such as time planning and consolidating integration relationships or the interaction between different disciplines / subjects to achieve a better integration in the project.

Keywords: Project-based learning, multidisciplinary, competences

\section{INTRODUCCIÓN}

La resolución de problemas requiere algo más que memorizar información (Cano \& Salazar, 2011). El aprendizaje se sustenta en la experiencia de enfrentarse a escenarios diferentes donde los alumnos puedan aplicar sus conocimientos.
Por tanto, el conocimiento significativo no se puede alcanzar con una simple memorización de datos desvinculados entre sí.

ABP es una herramienta metodológica ampliamente utilizada, tanto en la educación superior como universitaria (Sánchez, 2013), donde el alumno planifica y gestiona su propio proyecto teniendo como resultado una actividad observable. ABP articulan un amplio número de actividades formativas (Aguirregabiria Barturen \& García Olalla, 2020), como la formación de equipos con miembros de diferentes, áreas disciplinares, profesiones, idiomas y culturas que trabajan juntos para realizar proyectos con el propósito de solucionar problemas reales (Reverte Bernabeu et al., 2007). El ABP también implica el desarrollo de habilidades como la colaboración, planificación, comunicación, toma de decisiones y gestión del tiempo (Dickinson et al., 1998), y de competencias como pensamiento crítico y práctico mediante la integración de la teoría y la práctica (Lorente, 2017). Según la bibliografía, el ABP al utilizar casos reales mejora la motivación, medida por un incremento de la mayor asistencia y participación del alumnado (Bottoms \& Webb, 1998), y por tanto un aumento en el nivel de satisfacción al ver que la utilidad práctica del aprendizaje (Willard \& Duffrin, 2003). Esta mayor motivación y satisfacción también se ha visto reflejado en una mejora de la calificación final y el aprendizaje autónomo, así como la prolongación en el tiempo de lo aprendido (Mioduser \& Betzer, 2008).

Por otro lado, del profesor requiere un papel muy diferente al que estamos acostumbrados. El profesor debe convertirse en un guía que estimule a los estudiantes a aprender a aprender. El profesor busca soluciones a problemas, genera preguntas, diseña planes, recopila datos, establece conclusiones (Ayerbe Lopez \& Perales Palacios, 2020), pero para alcanzar el objetivo marcado es necesario un correcto diseño del proyecto, con unas metas claras y concisas (Helle et al., 2006). Y para que el diseño sea correcto, el proyecto no debe ser el colofón a una determinada unidad didáctica, sino la columna vertebral por la que transcurre el proceso enseñanza-aprendizaje (Larmer \& Mergendoller, 2010). Los proyectos planteados no deben ser excesivamente complejos, que puedan desencadenar a una pérdida de motivación por parte del estudiante (Ayerbe Lopez \& Perales Palacios, 2020). 
Tradicionalmente, el ABP es una metodología de aprendizaje utilizada en el campo de la medicina desde los años 60, pero que poco a poco se ha ido expandiendo a otros campos de la enseñanza como en la ingeniería (Mills \& Treagust, 2003). Por ejemplo, Hassan et al. (2008) implementó ABP con éxito en cursos de ingeniería electrónica, o en Australia, Stewart (2007) observó que los alumnos de posgrado con habilidades de aprendizaje autorregulado estaban mejor preparados para finalizar con éxito los proyectos planteados. Pero no únicamente, se han utilizado en programas de ingeniería, sino también en el campo de la lingüística (Gibbes \& Carson, 2014).

Por todo ello, el aprendizaje basado en proyectos colaborativos puede ser de gran utilidad para preparar a los estudiantes a hacer frente a problemas reales, generalmente multidisciplinares, fomentando el trabajo en equipo y las competencias necesarias para tener un mayor éxito laboral.

\section{CONTEXTO}

La innovación docente se aplicó en el Máster Oficial interuniversitario de acuicultura (MOIA), por tanto, en el ámbito de educación superior de posgrado. MOIA es un Máster de un año de duración, de cuya docencia se encargan la Universitat Politécnica de València (UPV) junto con la Universitat de València (UV) y con el Instituto de Acuicultura de Torre de la Sal (Centro Superior de Investigaciones Científicas; CSIC). La medía de alumnos suele ser de 25 y su perfil muy variado. Hay alumnos procedentes de titulaciones científico-técnicas (grado en biología, ingeniería agronómica, veterinaria, farmacia, ciencias del mar, ingeniería de montes, biotecnología) o bien otras titulaciones procedentes de Sudamérica, como ingeniería zootecnista o ingeniería pesquera.

Desde el curso 2006/2007 que se imparte el MOIA (Universitat Politècnica de Valencia, Máster Universitario en Acuicultura, titulaciones), siempre ha incluido asignaturas con un marcado enfoque práctico, como "Diseño y gestión de piscifactorías" o "Ingeniería de sistemas", "Nutrición y alimentación de especies acuícolas" o "Producción de especies continentales y tropicales". Dada la naturaleza de estas asignaturas, la metodología docente de estas asignaturas siempre ha planteado temáticas lo más cercanas posibles al contexto profesional. Es por ello, que las asignaturas tienen una patente relación entre ellas. Sin embargo, se ha observado que los alumnos tienen una visión individualizada de las asignaturas, limitándose a aprobarlas sin llegar a un aprendizaje multidisciplinar. Este hecho, limita la posibilidad de obtener una visión más real y general de las situaciones y problemáticas reales en el sector acuícola.

Con el fin de solucionar esta problemática se plantea implantar el ABP en una asignatura del último cuatrimestre: Diseño y Gestión de instalaciones piscícolas. Esta asignatura permite englobar todas estas disciplinas y asignaturas, ya que dentro de la realización del proyecto de las asignatura considera aspectos tanto desde el punto de vista de diseño como nutricional, de biología y zootecnia de las diferentes especies acuícolas, económico, de ingeniería de las instalaciones, manejo de especies y de piscifactorías, impacto ambiental, bienestar de los animales,...De modo que se pueden aplicar todas estas disciplinas mediante el desarrollo de un proyecto, pudiendo adquirir una visión en conjunto de las asignaturas impartidas y la conexión entre ellas
Por otro lado, la sociedad demanda que la docencia universitaria potencie y facilite la inserción socio-laboral de los futuros egresados. Para ello, el desarrollo de las competencias es un elemento que cada día es más apreciado por los empleadores, siendo de especial relevancia las relacionadas con el pensamiento crítico o práctico (Yaoxiang, 2019). Con este fin, la inclusión de metodologías activas como el aprendizaje basado en proyectos (ABP) permite un rol más activo por parte del alumno, siguiendo la línea marcada por el Espacio Europeo de Educación Superior (EEES), que marca una metodología docente centrada en el alumno (Aguirregabiria Barturen \& García Olalla, 2020).

En concreto, gracias a la implantación de ABP se pretende potenciar las competencias de aplicación y pensamiento práctico, análisis y resolución de problemas, innovación, creatividad y emprendimiento, diseño y proyecto, comunicación efectiva, trabajo en equipo, así como planificación y gestión del tiempo.

Las hipótesis planteadas son las siguientes:

- La incorporación de ABP basado en el desarrollo de una piscifactoría permitirá al alumno una integración de los conceptos aprendidos en asignaturas previas

- La resolución de problemas desde una perspectiva cercana al mundo laboral estimulará la participación del alumno en las actividades propuestas

- La innovación docente planteada, ABP, mejorará la adquisición de las competencias.

- Alumnos con unas mejores competencias tendrán una inserción laboral con mayor éxito.

Con el fin de validar las hipótesis, se plantearon los siguientes objetivos:

1. Alcanzar un aprendizaje multidisciplinar de las materias impartidas.

2. Mejorar en la adquisición de competencias.

3. Aprender a manejar recursos TIC para finalizar con éxito el proyecto.

4. Mejorar la motivación y participación del alumnado en las actividades

\section{DESCRIPCIÓN}

El ABPr se implantó en la asignatura Diseño y Gestión de instalaciones piscícolas, donde los alumnos deben realizar el diseño de una piscifactoría: desde la planificación zootécnica, dimensionando instalación y los equipos, y teniendo en cuenta aspectos medioambientales (generación de residuos y consumo de agua) y económicos. En la Figura 1 se muestra un esquema general de las diferentes tareas a desarrollar. La Tarea 1 consiste en la recopilación de las asignaturas/conocimientos adquiridos en previas asignaturas y que le serán necesarias para llevar a cabo el proyecto. Para ello lo alumnos entregan un informe de una hoja de extensión, describiendo los conocimientos previos adquiridos en otras asignaturas. 


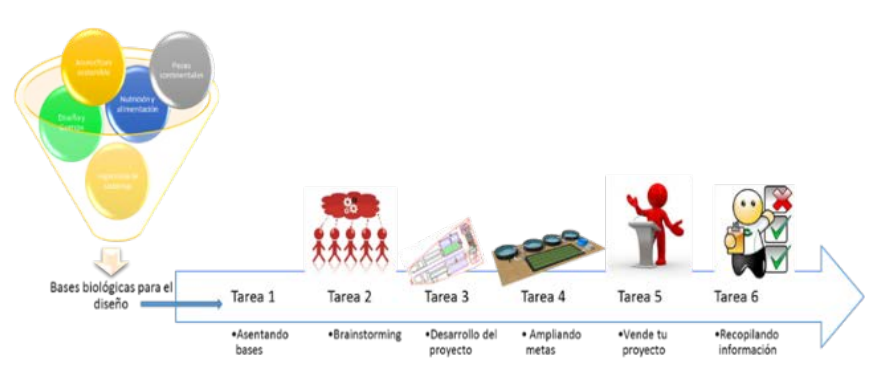

Figura 1. Secuencia de las tareas a desarrollar

Seguidamente se realizó una actividad grupal de 2 o 3 alumnos ("Brainstorming", Tarea 2). Con esta actividad se pretendía fomentar la participación y la integración de todos los miembros del grupo, estableciéndose los siguientes objetivos:

1. ¿Qué especie vamos a elegir para diseñar la piscifactoría?

2. ¿Qué sistema de producción es el más indicado para esta especie?

3. ¿Hasta dónde podemos llegar en el proyecto con los conocimientos adquiridos previamente?

4. ¿Qué información hay disponible que nos permita poder desarrollar el proyecto?

Al finalizar el brainstorminig, los alumnos deben entregar las respuestas al profesor a cada una de las preguntas.

El desarrollo del proyecto se efectuó dentro de la Tarea 3 y 4 (Figura 1), en la cual los alumnos utilizando hojas de cálculo debían realizar la planificación de la producción y diseño zootécnico de la piscifactoría. La hoja de cálculo servirá como indicador de consecución de esta tarea. El diseño debía seguir la metodología descrita en Jover et al. (2016). Como material de apoyo, los alumnos disponían de videos explicativos de 10 minutos para los conceptos umbrales más complicados.

Una vez finalizado el proyecto de la instalación, los alumnos deben exponer su proyecto (Tarea 5: "Vende tu proyecto") destacando las ventajas de su proyecto, tanto productivas, como económicas, y será necesario una calificación superior a 5 para considerar que se ha cumplido con los objetivos de esta tarea. Finalmente, con el fin de evaluar el grado de satisfacción de los alumnos del ABPr implantado (Tarea 6. Recopilando información, Figura 1), se realizó una encuesta (Tabla 1).

Tabla 1. Cuestionario de evaluación de los alumnos sobre la metodología docente: $\mathrm{ABPr}$

\begin{tabular}{ll}
\hline \multicolumn{1}{c}{ Cuestionario sobre el ABPr } & Escala \\
\hline Q1 ¿Cree usted qué el proyecto plateado es & \\
importante para su formación profesional? & \\
Q2 ¿Se aplicaron los conceptos vistos en clase para el & \\
desarrollo del proyecto de aula? \\
Q3 ¿Se aplicaron los conceptos vistos en asignaturas \\
previas para el desarrollo del proyecto de aula? \\
Q4¿Este tipo de metodologías para el aprendizaje \\
satisfacen sus expectativas como estudiante \\
comparándola con otros métodos tradicionales? \\
Q5¿Considera que esta metodología es útil para \\
aplicar los conocimientos vistos en clase para \\
solucionar problemas del proyecto?
\end{tabular}

Q6 Usted considera que la orientación para el

desarrollo del trabajo por parte del docente cumplió

con sus expectativas

También se realizó una encuesta de valoración sobre las competencias desarrolladas gracias a ABP (Tabla 2).

Tabla 2. Cuestionario de evaluación de las competencias trabajadas durante el ABPr

\begin{tabular}{|c|c|}
\hline Cuestionario sobre el ABPr & Escala \\
\hline $\begin{array}{l}\text { Consideras que las estrategias } \mathrm{ABPr} \text { son muy } \\
\text { adecuadas para desarrollar (entre otras) las siguientes } \\
\text { capacidades (cada ítem) }\end{array}$ & \\
\hline CA. Trabajo en grupo & \\
\hline CB. Aprendizaje autónomo & $1-5$ \\
\hline $\begin{array}{l}\text { CC. Participar activamente en el desarrollo del } \\
\text { proyecto }\end{array}$ & \\
\hline CD. Capacidad para Planificación el tiempo & \\
\hline CE. Capacidad de expresarse de forma adecuada & \\
\hline $\begin{array}{c}\text { Otros aspectos que le gustaría destacar tanto } \\
\text { negativos como positivos }\end{array}$ & $\begin{array}{c}\text { Respuesta } \\
\text { abierta }\end{array}$ \\
\hline
\end{tabular}

\section{Resultados}

En general, la valoración del ABPr fue alta (Figura 2), obteniendo una media general superior a 4 sobre 5. Las cuestiones mejor valoradas fueron las Q1 y Q6 (Tabla 1), relativas a la importancia del desarrollo del proyecto para su formación profesional y a las expectativas del alumno por la asignatura. Así también, los alumnos han valorado muy positivamente los conocimientos impartidos en clase y desarrollados en el proyecto (respuesta Q2). No obstante, la respuesta que presentó significativamente una menor puntuación fue la respuesta Q3. La posible explicación de la menor puntuación se puede encontrar en la respuesta abierta. Los alumnos reclamaron que se incluyeran en temas relacionados con la patología o de calidad del agua en el proyecto, necesarios para poder realizar un diseño de una piscifactoría adecuado. Sin embargo, la valoración de la metodología docente respecto a métodos tradicionales (respuesta Q4) y su utilidad para solucionar problemas reales (pensamiento práctico; respuesta Q5) fueron muy positivos (niveles mayores de 4 sobre 5).

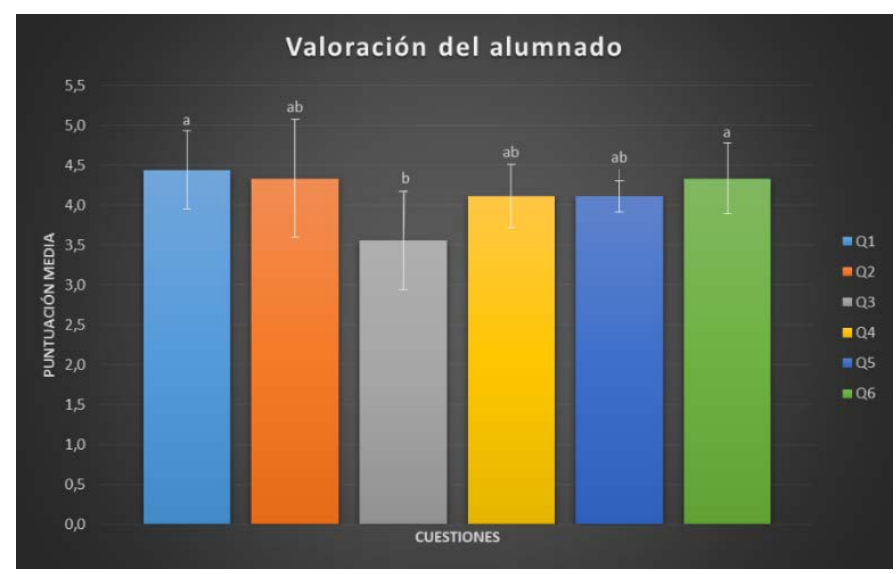

Figura 2. Valoración media de los alumnos de la ABPr*. Los datos que aparecen en el gráfico son la media \pm desviación estándar $(n=10)$. Letras diferentes en las barras indican 
diferencias estadísticas significativas con una $\mathrm{P}<0,05$. *Cuestiones planteadas se pueden consultar en la Tabla 1

En relación con el estímulo de los estudiantes en el desarrollo de habilidades para resolver situaciones reales, fue una de las metas más difíciles de alcanzar en el proyecto, principalmente por falta de tiempo necesario para la búsqueda de información. De hecho, uno de los aspectos con una valoración algo menor por parte del alumnado de la metodología docente fue capacidad para Planificación el tiempo (CD; Figura 3), lo cual posiblemente ha influido en la falta de tiempo para la búsqueda de información

Durante el desarrollo del proyecto se trabajó en grupo, fomentando las sinergias y trabajando diferentes competencias. Esto se evidencia con la excelente valoración de la capacidad adquirida para el trabajo en grupo y el trabajo autónomo. (Figura 3; respuestas CA y CB). Sin embargo, los alumnos han visto que el $\mathrm{ABPr}$, no ha contribuido considerablemente a adquirir una mayor capacidad de expresarse adecuadamente, debido a que el tiempo para trabajar esta capacidad fue reducido (CE).

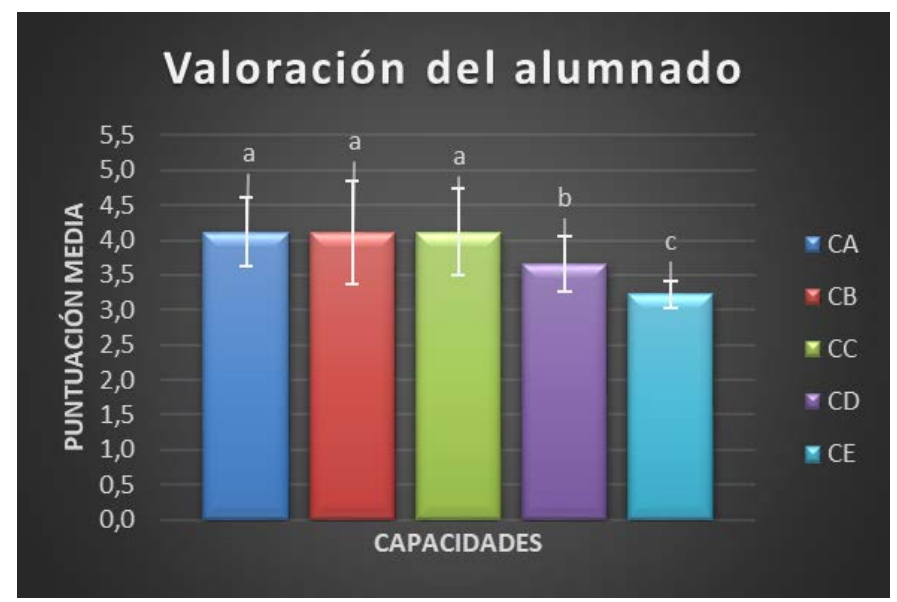

Figura 3. Valoración media de los alumnos de las competencias trabajadas gracias al ABPr*. Los datos que aparecen en el gráfico son la media \pm desviación estándar $(n=10)$. Letras diferentes en las barras indican diferencias estadísticas significativas con una $\mathrm{P}<0,05$.

Por lo tanto, en base a los resultados, los principales aspectos a mejorar podrían ser:

-Planificación del tiempo para desarrollar el proyecto. Posiblemente, el tiempo que tenían los alumnos para desarrollar un proyecto de esta magnitud era corto.

-Mejorar la multidisciplinidad. Aportar recursos de documentación o bibliográficos al inicio de la asignatura de aquellas materias que puedan ser útiles para el desarrollo del proyecto.

En este sentido, los alumnos echaron en falta la inclusión en el proyecto de materias relacionadas con patología o bien calidad del agua, que sería muy útil a la hora de estimar mortalidades y/o tratamientos para una especie piscícola.

Como puntos fuertes se deben de subrayar

-Seguimiento personalizado de los alumnos: La implantación de ABPr, permite un seguimiento muy preciso del proceso de aprendizaje de los grupos, así como una evaluación muy personalizada de todo el proceso.

-Elevado enfoque empresarial del proyecto. Debido a que el ABPr está enfocado al sector empresarial, el proyecto planteado se acerca mucho a situaciones reales en piscifactorías. Esta proximidad a la empresa motiva especialmente a los alumnos, que al constatar la aplicabilidad en el sector privado del trabajo desarrollado.

-Capacidad para aplicar tecnologías de la información y comunicación (TICs). Debido a que en la UPV disponemos de una plataforma para aplicar las TIC, éstas pueden facilitar una docencia más interactiva, pudiendo fomentar la comunicación e interacción entre alumnos y profesores.

-Contacto con el sector acuícola. Durante el desarrollo del proyecto, los alumnos tienen la posibilidad de ponerse en contacto con profesionales procedentes de sector y de centros de investigación de alto prestigio como el CSIC (Instituto de Acuicultura de Torre de la Sal) o el Instituto Español de Oceanografía (IEO) de Murcia, así como empresas del sector.

Cabría destacar que tras la implantación de ABPs, se registró por primera vez en esta asignatura un $100 \%$ de asistencia. Además, los resultados académicos han sido mejores que otros años, con una puntuación media de 8,05 respecto a 7,5 de los últimos 5 años.

\section{CONCLUSIONES}

Se puede concluir que la implantación del ABPs ha permitido un mayor desarrollo de las competencias, además de mejorar la participación del alumnado en las actividades propuestas, dando como resultado una mejor calificando académica final. No obstante, se deben de mejorar algunos aspectos como es la planificación del tiempo y consolidar relaciones de integración o la interacción entre diferentes disciplinas/asignaturas de manera que tengan una mejor integración en el proyecto.

\section{AgradeCIMIENTOS}

Agradecer a los alumnos su participación en las encuestas, y a los profesores por su esfuerzo en implantar esta nueva metodología docente. También nos gustaría agradecer a agradecer el apoyo del Vicerrectorados de Estudios, Calidad y Acreditación de la Universitat Politècnica de València al proyecto PIME/18-19/74 "Aprendizaje basado en proyectos para fomentar la visión profesional en el máster de Acuicultura" y al PIME/20-21/213 "Estrategias para favorecer la autorregulación en el aprendizaje de competencias".

\section{REFERENCIAS}

Aguirregabiria Barturen, J., \& García Olalla, A. M. (2020). Aprendizaje basado en proyectos y desarrollo sostenible en el Grado de Educación Primaria. Enseñanza de Las Ciencias, 38(2), 5-24.

Ayerbe Lopez, J., \& Perales Palacios, F. J. (2020). “ Reinventa tu ciudad": aprendizaje basado en proyectos para la mejora de la conciencia ambiental en estudiantes de Secundaria. Enseñanza de Las Ciencias, 38(2), 181-203.

Bottoms, G., \& Webb, L. D. (1998). Connecting the curriculum to" real life." Breaking Ranks: Making it happen. ERIC Clearinghouse.

Cano, J. M. M., \& Salazar, T. del N. J. M. (2011). Aprendizaje 
con base en proyectos para desarrollar capacidades de problematización en educacion superior/Project-based learning to develop problematization skills in higher education. Actualidades Investigativas En Educación, 11(1).

Dickinson, K. P., Soukamneuth, S., Yu, H. C., Kimball, M., D’amico, R., Perry, R., Kingsley, C., \& Curan, S. P. (1998). Providing Educational Services in the Summer Youth Employment and Training Program. Technical Assistance Guide.

Gibbes, M., \& Carson, L. (2014). Project-based language learning: an activity theory analysis. Innovation in Language Learning and Teaching, 8(2), 171-189.

Hassan, H., Martinez, D. J., Peres, A., Albaladejo, J., \& Capella, J. (2008). Integrated multicourse project based learning in electronic engineering. The International Journal of Engineering Education, 24(3), 581-591.

Helle, L., Tynjälä, P., \& Olkinuora, E. (2006). Project-based learning in post-secondary education-theory, practice and rubber sling shots. Higher Education, 51(2), 287314.

Larmer, J., \& Mergendoller, J. R. (2010). Seven essentials for project-based learning. Educational Leadership, 68(1), 34-37.

Lorente, E. (2017). El trabajo por proyectos en Primaria: dos experiencias, dos mundos. Universitat Jaume I.

Mills, J. E., \& Treagust, D. F. (2003). Engineering educationIs problem-based or project-based learning the answer. Australasian Journal of Engineering Education, 3(2), 2-
16.

Mioduser, D., \& Betzer, N. (2008). The contribution of Projectbased-learning to high-achievers' acquisition of technological knowledge and skills. International Journal of Technology and Design Education, 18(1), 5977.

Reverte Bernabeu, J., Gallego Sánchez, A. J., Molina-Carmona, R., \& Satorre Cuerda, R. (2007). El aprendizaje basado en proyectos como modelo docente. Experiencia interdisciplinar y herramientas groupware.

Sánchez, J. (2013). Qué dicen los estudios sobre el Aprendizaje Basado en Proyectos. Actualidad Pedagógica.

Stewart, R. A. (2007). Investigating the link between self directed learning readiness and project-based learning outcomes: the case of international Masters students in an engineering management course. European Journal of Engineering Education, 32(4), 453-465.

Universitat Politècnica de Valencia, Máster Universitario en Acuicultura, titulaciones. https://www.upv.es/titulaciones/MUA/indexc.html.

Willard, K., \& Duffrin, M. W. (2003). Utilizing project-based learning and competition to develop student skills and interest in producing quality food items. Journal of Food Science Education, 2(4), 69-73.

Yaoxiang, L. (2019). Practical Thinking and Innovative Strategy of Diagnosing and Improving Teaching Work in Higher Vocational Colleges. Advances in Educational Technology and Psychology, 3(1), 80-88. 